\title{
Influence of Plant Growth Regulators on shoot and root length of Fagopyrum esculentum Moench of Himalayan Region
}

\author{
Komal Jamwal ${ }^{1 *}$, Sujata Bhattacharya ${ }^{1}$, Sunil Puri ${ }^{1}$ and Dushyant Sharma ${ }^{2}$
}

${ }^{1}$ Shoolini University of Biotechnology and Management Sciences; Near Head P.O., The Mall Solan, H.P. (173 212 ), India ${ }^{2}$ Dr. Y. S. Parmar University of Horticulture and Forestry, College of Horticulture and Forestry, Neri,

Distt. Hamirpur, H.P. (177 001), India

\section{Corresponding Author}

Komal Jamwal

e-mail: komaljamwal72@gmail.com

\author{
Article History \\ Article ID: IJEP0375 \\ Received in 29 $9^{\text {th }}$ June, 2020 \\ Received in revised form $12^{\text {th }}$ July, 2020 \\ Accepted in final form $22^{\text {nd }}$ July, 2020
}

\begin{abstract}
Present investigation was made to evaluate effect of PGRs on shoot and root length of Fagopyrum esculentum. Results revealed that there was increase in shoot length by GA at $100 \mathrm{mg} \mathrm{l}^{-1}$ and root length by IAA at $50 \mathrm{mg} \mathrm{l}^{-1}$ at 30,60 and 90 days of plant growth. ABA at $25 \mathrm{mg} \mathrm{l}^{-1}$ and $100 \mathrm{mg} \mathrm{l}^{-1}$ decreased shoot length as well root length. BAP at $100 \mathrm{mg} \mathrm{l}^{-1}$ also decreased root length. In combinations, IAA+GA $100 \mathrm{mg}$ $\mathrm{I}^{-1}$ treatment enhanced shoot length as well as root length. Decrease in shoot length was observed in IAA+ABA, ABA+BAP (50 and 100 mg $\left.\mathrm{I}^{-1}\right)$ and root length by ABA+BAP $\left(100 \mathrm{mg}^{-1}\right)$ treatment.
\end{abstract}

Keywords: Antioxidant activity, Fagopyrum esculentum, food, phenolic compounds

\section{Introduction}

It is one of the essential unattended crops and is grown as a minor grain crop in the Indian Himalayas, particularly in the high-altitude areas (Sharma et al., 2018). Due to its frost resistance, short growth period and easy cultivation, buckwheat is common in high-altitude areas at $2000 \mathrm{~m}$ and in Tibet it is found at elevations of up to $4500 \mathrm{~m}$ (Zhang et al., 2012). It is a dicotyledonous, multipurpose, summergrowing annual plant of family Polygonaceae with knotted stem of 30-60 in height, with shallow root system. Many of the health benefits of buckwheat have been attributed to its high levels of phenolic compounds and antioxidant activity (Wijngaard and Arendt, 2006). Common buckwheat is rich in vitamins, especially those of the B group (Fabjan et al., 2003) and is significant source of microelements ( $\mathrm{Zn}, \mathrm{Cu}, \mathrm{Mn}, \mathrm{Se}$ ) as well as macroelements (K, Na, Ca, Mg) (Stibilj et al., 2004). Major flavonoids in buckwheat are rutin, quercetin, orientin, homoorientin, vitexin, isovitexin, hyperin and catechins (Morishita et al., 2007). It has been reported that the ethyl acetate and ethanol extracts of the stem, seed and aerial parts of buckwheat show neuroprotective effect through acetylcholinesterase, butyrylcholinesterase and tyrosinase inhibitory and antioxidant activity (Gulpinar et al., 2012).

Plant growth regulators (PGRs) are invention of agrochemicals subsequent to pesticides and fertilizers. PGRs improve growth of plants by influencing their usual homeostasis; mainly the hormonal regulation (Ahmada et al., 2019). Number of studies is reported where PGRs enhanced shoot length (Sumathi et al., 2017; Khunte et al., 2020), root length (Amri, 2011; Galavi et al., 2013). F. esculentum has enormous, medicinal, nutritional and economic value and if promoted, could highly contribute to reduction of poverty mainly in rural areas and to the improvement of both nutritional and health status of the local populations. Till date, there are no reports regarding the effects of PGRs on common buckwheat. Keeping these facts in mind, present study has been undertaken to see the relative effect of $F$. esculentum to plant growth regulators.

\section{Materials and Methods}

The seeds of Fagopyrum esculentum Moench were obtained from Himachal Pradesh Agricultural University, Research Station, Sangla, Kinnaur (HP). Experiments were conducted in the laboratory and nursery area of Shoolini University of Biotechnology and Management Sciences, Solan.

\subsection{Seedling growth assays and growing conditions}

Seeds of Fagopyrum esculentum selected for uniformity, damaged and insect infected seeds were discarded and the hollow ones were rejected by floating method in distilled water. Surface sterilization of seeds was done with $0.1 \%$ $\mathrm{HgCl}_{2}$ prior to sowing, after which the seeds were rinsed 
three times with distilled water. Seeds were sown in the nursery beds, in the Herbal Garden of Shoolini University, Solan (Latitude $30^{\circ} 51^{\prime} \mathrm{N}$, longitude $77^{\circ} 07^{\prime} \mathrm{E}$ and altitude 1195 $\mathrm{m})$, where the average annual rainfall was $1315.6 \mathrm{~mm}$. The average maximum and minimum temperatures were $32^{\circ} \mathrm{C}$ and $2^{\circ} \mathrm{C}$, respectively. Nursery beds were watered regularly. When the first leaf appeared the seedlings were transferred to pots (20 cm diameter). The pots were filled with $3 \mathrm{~kg}$ uniform soil mixture containing soil: sand: farm yard manure (FYM) in 1:1:1 ratio. Three seedlings per pot in replicates of three were used for each treatment. No inorganic fertilizer and systemic pesticide were used during the experiment. The pots were arranged in a entirely randomized design and the locations of the pots were changed weekly to avoid position effect.

\subsection{Treatments with plant growth regulators}

Plant growth regulators spray was done after one week of transplanting the plants to pots. Four major hormones: indole Acetic Acid (IAA), benzylaminopurine (BAP), abscisic Acid (ABA) and gibberellic Acid (GA), were used solely as well as in combinations i.e. IAA+BAP, IAA+ABA, IAA+GA, $A B A+B A P$, $B A P+G A$ and $A B A+G A$ in concentration of 25,50 and $100 \mathrm{mg}$ $\mathrm{I}^{-1}$ through foliar spray. Shoot length $(\mathrm{cm})$ and Root length (cm) were measured at different growth stages i.e., 30, 60 and 90 days.

\subsection{Statistical analysis}

The data was analyzed statistically using Graph Pad Prism ${ }^{\circledR}$ 5.2. Mean values were calculated from measurements of three replicates and the standard error of means were determined. Two-way analysis of variance (ANOVA) was applied to determine the significance of results between different treatments and Bonferroni's post tests were performed at the significance level of $p<0.05$.

\section{Results and Discussion}

\subsection{Shoot length}

As expected progressive increase in shoot length was

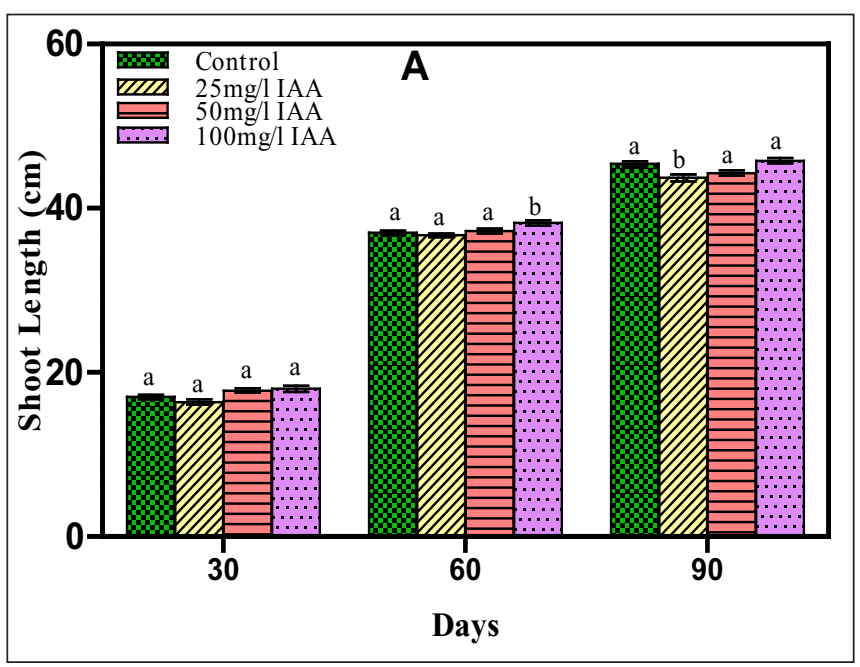

noted with an increase in time period. GA at $100 \mathrm{mg} \mathrm{I}^{-1}$ concentration gave highest shoot length at 30 days $(20.50$ $\mathrm{cm}, 20.5 \%$ increase from control), at 60 days $(42.30 \mathrm{~cm}$, $14.32 \%$ increase from control) and at 90 days $(54.60 \mathrm{~cm}$, $20.26 \%$ increase from control) of plant growth. However, at 30 days ABA $25 \mathrm{mg} \mathrm{l}^{-1}(14.30 \mathrm{~cm}, 15.9 \%$ decrease from control), at 60 days $A B A 100 \mathrm{mg} \mathrm{l}^{-1}(34.30 \mathrm{~cm}, 7.3 \%$ decrease from control) and at 90 days ABA $25 \mathrm{mg} \mathrm{l}^{-1}(41.80 \mathrm{~cm}, 7.9 \%$ decrease from control) treated plants resulted in minimum shoot length (Figure 1). Combination of phytohormones produced, maximum shoot length at 30 days $(34 \mathrm{~cm}, 100 \%$ increase from control), at 60 days $(46 \mathrm{~cm}, 24.3 \%$ increase from control) and at 90 days $(58 \mathrm{~cm}, 27.8 \%$ increase from control) as shown by IAA+GA $100 \mathrm{mg} \mathrm{l}^{-1}$ treated plants. Minimum shoot length at 30 days was seen in IAA+ABA $100 \mathrm{mg} \mathrm{l}^{-1}$ treated plants $(13.50 \mathrm{~cm}, 20.6 \%$ decrease from control), at 60 days in ABA+BAP $100 \mathrm{mg} \mathrm{l}^{-1}$ treated plants (30.20 cm, $18.4 \%$ decrease from control) and at 90 days in ABA+BAP $50 \mathrm{mg} \mathrm{l}^{-1}$ treated plants $(35.20 \mathrm{~cm}, 22.5 \%$ decrease from control) (Figure 1).

At 30 days of growth IAA showed no variability from control (Figure 1.1 A) while ABA had reduced growth (Figure 1.1 C) and GA enhanced growth (Figure 1.1 D). Combination of growth regulators revealed synergistic effects in case of IAA+BAP and IAA+GA (Figure $1.1 \mathrm{G}$ and I); while combination with $A B A$ produced retarded effects (Figure $1 \mathrm{~F}$ and $\mathrm{H}$ ). It is evident from Bonferroni post-test (Table 1) that after 90 days untreated (control) plants showed significant difference $(p<0.05)$ with all the three concentrations $\left(25,50\right.$ and $\left.100 \mathrm{mg} \mathrm{l}^{-1}\right)$ of BAP, ABA and $G A$. Combination of $I A A+G A$ and $A B A+B A P$ also showed a significant difference. IAA (25 mg l-1, $\left.50 \mathrm{mg} \mathrm{l}^{-1}\right)$ and GA (25 $\mathrm{mg} \mathrm{l}^{-1}, 50 \mathrm{mgl}^{-1}$ ) revealed significant differences with IAA (100 $\left.\mathrm{mg} \mathrm{l}^{-1}\right)$ and $\mathrm{GA}\left(100 \mathrm{mg} \mathrm{l}^{-1}\right)$ treated plants, respectively. Plants given treatment of $25 \mathrm{mg} \mathrm{I}^{-1} \mathrm{IAA}+\mathrm{BAP}, \mathrm{IAA}+\mathrm{GA}, \mathrm{ABA}+\mathrm{BAP}$ also showed significant difference with $50 \mathrm{mg} \mathrm{l}^{-1}$ and $100 \mathrm{mg}$ $\mathrm{I}^{-1}$ of IAA+BAP, IAA+GA, ABA+BAP treated plants. $25 \mathrm{mg} \mathrm{l}^{-1}$ concentration of $B A P+G A$ and $A B A+G A$ showed significant difference with $100 \mathrm{mg} \mathrm{l}^{-1}$ concentration of $B A P+G A$ and

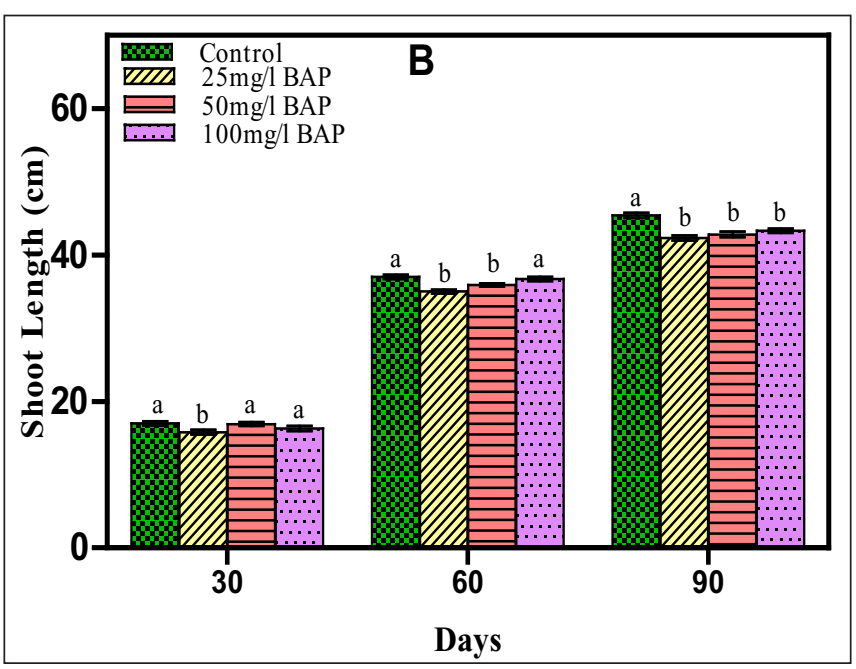

Figure 1: Continue... 

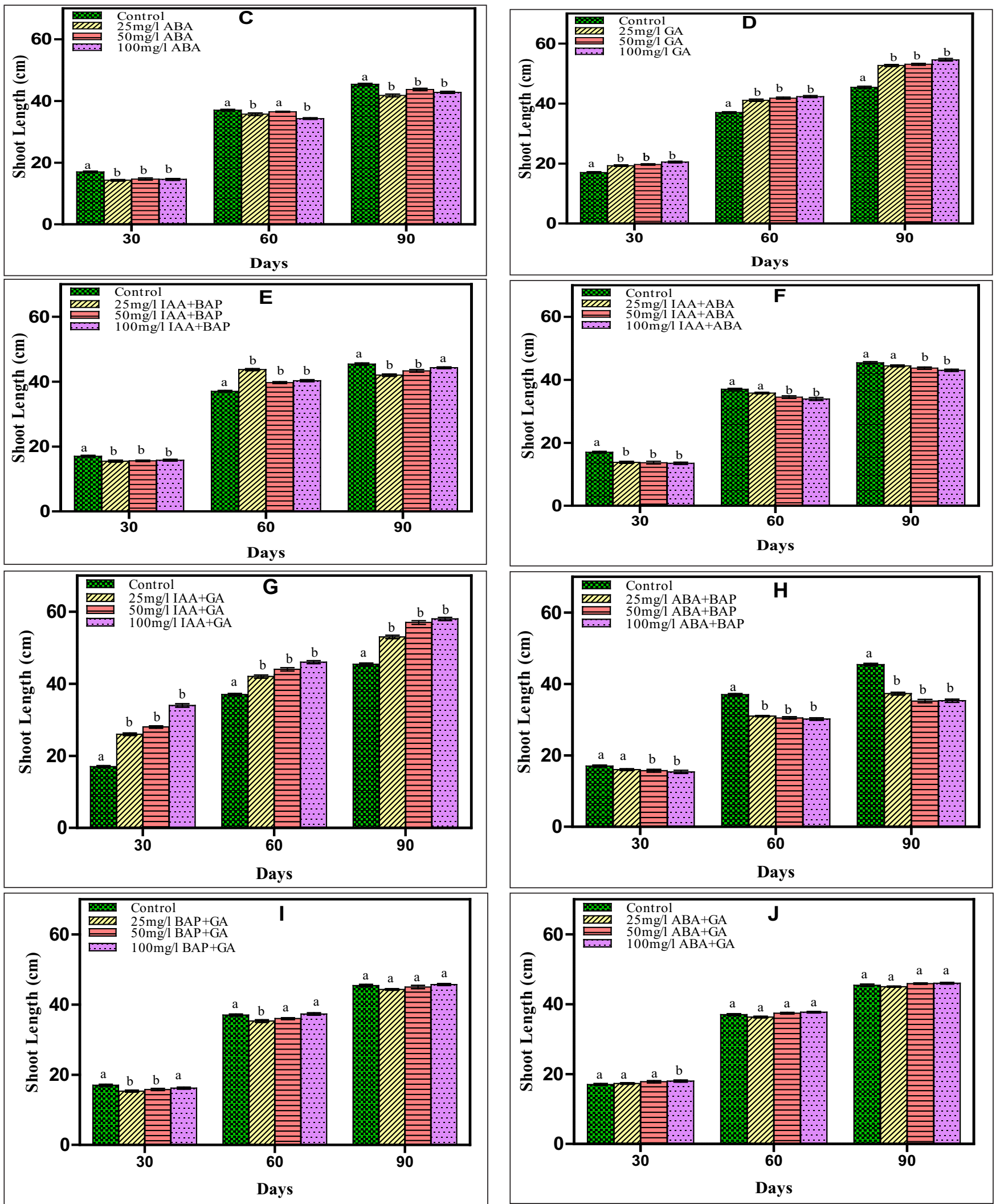

Figure 1: Shoot length in Fagopyrum esculentum on different days treated with IAA (A), BAP (B), ABA (C), GA (D), IAA+BAP (E), $I A A+A B A(F), I A A+G A(G), A B A+B A P(H), B A P+G A(I)$ and $A B A+G A(J)$. Values are mean $\pm S E ; n=3$, analysed by Two-way ANOVA followed by Bonferroni's multiple comparison test. Values followed by the same letter are not statistically different $(p<0.05)$ compared with control 
Table 1: Bonferroni post-test for comparison between treated and untreated plants of Fagopyrum esculentum for shoot length

\begin{tabular}{|c|c|c|c|c|c|c|c|c|c|c|c|c|}
\hline \multirow[t]{2}{*}{ Days } & \multicolumn{6}{|c|}{ IAA } & \multicolumn{6}{|c|}{ BAP } \\
\hline & $\begin{array}{c}\text { Control } \\
\text { vs } 25 \\
\mathrm{mg} \mathrm{I}^{-1} \\
\text { IAA }\end{array}$ & $\begin{array}{c}\text { Control } \\
\text { vs } 50 \\
\mathrm{mg} \mathrm{l}^{-1} \\
\text { IAA }\end{array}$ & $\begin{array}{c}\text { Control } \\
\text { vs } 100 \\
\mathrm{mg} \mathrm{l}^{-1} \\
\text { IAA }\end{array}$ & $\begin{array}{l}25 \mathrm{mg} / \mathrm{I} \\
\mathrm{IAA} \mathrm{Vs} \\
50 \mathrm{mg} \\
\mathrm{I}^{-1} \mathrm{IAA}\end{array}$ & $\begin{array}{c}25 \mathrm{mg} \mathrm{l}^{-1} \\
\text { IAA Vs } \\
100 \mathrm{mg} \\
\mathrm{I}^{-1} \mathrm{IAA}\end{array}$ & $\begin{array}{c}50 \mathrm{mg} \mathrm{l}^{-1} \\
\mathrm{IAA} \mathrm{Vs} \\
100 \mathrm{mg} \\
\mathrm{I}^{-1} \mathrm{IAA}\end{array}$ & $\begin{array}{l}\text { Control } \\
\text { vs } 25 \\
\mathrm{mg} \mathrm{l}^{-1} \\
\text { BAP }\end{array}$ & $\begin{array}{c}\text { Control } \\
\text { vs } 50 \\
\text { mg l-1 }^{-1} \\
\text { BAP }\end{array}$ & $\begin{array}{l}\text { Control } \\
\text { vs } 100 \\
\mathrm{mg} \mathrm{l}^{-1} \\
\text { BAP }\end{array}$ & $\begin{array}{c}25 \mathrm{mg} \mathrm{l}^{-1} \\
\text { BAP vs } \\
50 \mathrm{mg} \mathrm{l}^{-1} \\
\text { BAP }\end{array}$ & $\begin{array}{l}25 \mathrm{mg} / \mathrm{l} \\
\text { BAP vs } \\
100 \mathrm{mg} \\
\mathrm{I}^{-1} \mathrm{BAP}\end{array}$ & $\begin{array}{c}50 \mathrm{mg} \mathrm{l}^{-1} \\
\text { BAP vs } \\
100 \mathrm{mg} \\
\mathrm{I}^{-1} \text { BAP }\end{array}$ \\
\hline 30 & ns & ns & ns & $* *$ & $* *$ & ns & $*$ & ns & ns & $*$ & ns & ns \\
\hline 60 & ns & ns & $*$ & ns & $* *$ & ns & $* *$ & $*$ & ns & ns & $*$ & ns \\
\hline 90 & $* *$ & ns & ns & ns & $*$ & $* *$ & $* *$ & $*$ & $*$ & ns & ns & ns \\
\hline
\end{tabular}

Table Continue...

\begin{tabular}{|c|c|c|c|c|c|c|c|c|c|c|}
\hline \multirow[t]{2}{*}{ Days } & \multicolumn{8}{|c|}{ ABA } & \multicolumn{2}{|c|}{ GA } \\
\hline & $\begin{array}{c}\text { Control } \\
\text { vs } 25 \mathrm{mg} \mathrm{l}^{-1} \\
\text { ABA }\end{array}$ & \multicolumn{2}{|c|}{$\begin{array}{c}\text { Control } \\
\text { vs } 50 \mathrm{mg} \mathrm{l}^{-1} \\
\text { ABA }\end{array}$} & $\begin{array}{c}\text { Control } \\
\text { vs } 100 \mathrm{mg} \\
\mathrm{I}^{-1} \mathrm{ABA} \\
\end{array}$ & $\begin{array}{c}25 \mathrm{mg} \mathrm{l}^{-1} \mathrm{ABA}^{-} \\
\text {vs } 50 \mathrm{mg} \mathrm{l}^{-1} \\
\text { ABA }\end{array}$ & \multicolumn{2}{|c|}{$\begin{array}{c}25 \mathrm{mg} \mathrm{l}^{-1} \\
\text { ABA Vs } 100 \\
\mathrm{mg} \mathrm{l}^{-1} \mathrm{ABA}\end{array}$} & $\begin{array}{c}50 \mathrm{mg} \mathrm{l}^{-1} \\
\text { ABA Vs } 100 \\
\mathrm{mg} \mathrm{l}^{-1} \mathrm{ABA}\end{array}$ & $\begin{array}{c}\text { Control } \\
\text { Vs } 25 \mathrm{mg} \\
\mathrm{I}^{-1} \mathrm{GA}\end{array}$ & $\begin{array}{l}\text { Control } \\
\text { Vs } 50 \mathrm{mg} \\
\mathrm{I}^{-1} \mathrm{GA}\end{array}$ \\
\hline 30 & $* *$ & & $* *$ & $* *$ & ns & & רs & ns & $* *$ & $*$ \\
\hline 60 & $*$ & & ns & $* *$ & ns & & $*$ & $* *$ & $* *$ & $* *$ \\
\hline 90 & $* *$ & & $* *$ & $* *$ & $*$ & & רs & ns & $* *$ & $* *$ \\
\hline \multicolumn{11}{|c|}{ Table Continue... } \\
\hline \multirow[t]{2}{*}{ Days } & \multicolumn{4}{|c|}{ GA } & \multicolumn{6}{|c|}{$\mathrm{IAA}+\mathrm{BAP}$} \\
\hline & $\begin{array}{l}\text { Control } \\
\text { vs } 100 \\
\mathrm{mg} \mathrm{l}^{-1} \\
\mathrm{GA}\end{array}$ & $\begin{array}{l}25 \mathrm{mg} \\
{ }^{-1} \mathrm{GA} \mathrm{Vs} \\
50 \mathrm{mg} \\
\mathrm{I}^{-1} \mathrm{GA}\end{array}$ & $\begin{array}{c}25 \mathrm{mg} \mathrm{l}^{-1} \\
\mathrm{GA} \mathrm{Vs} \\
100 \mathrm{mg} \\
\mathrm{I}^{-1} \mathrm{GA}\end{array}$ & $\begin{array}{c}50 \mathrm{mg} \\
\mathrm{I}^{-1} \mathrm{GA} \text { vs } \\
100 \mathrm{mg} \\
\mathrm{I}^{-1} \mathrm{GA}\end{array}$ & $\begin{array}{l}\text { Control vs } \\
25 \mathrm{mg} \mathrm{l}^{-1} \\
\text { IAA+BAP }\end{array}$ & $\begin{array}{l}\text { Control } \\
\text { vs } 50 \\
\mathrm{mg} \mathrm{l}^{-1} \\
\text { AA+BAP }\end{array}$ & $\begin{array}{c}\text { Control } \\
\text { vs } 100 \\
\mathrm{mg} \mathrm{I}^{-1} \\
\text { IAA+BAP }\end{array}$ & $\begin{array}{c}25 \mathrm{mg} \mathrm{l}^{-1} \\
\text { IAA+BAP } \\
\text { vs } 50 \mathrm{mg} \mathrm{l}^{-1} \\
\text { IAA+BAP }\end{array}$ & $\begin{array}{c}25 \mathrm{mg} \mathrm{l}^{-1} \\
\text { IAA+BAP } \\
\text { vs } 100 \mathrm{mg} \\
\mathrm{I}^{-1} \text { IAA+BAP }\end{array}$ & $\begin{array}{c}50 \mathrm{mg} \mathrm{l}^{-1} \\
\text { IAA+BAP } \\
\text { vs } 100 \mathrm{mg} \\
\mathrm{I}^{-1} \text { IAA+BAP }\end{array}$ \\
\hline 30 & $* *$ & ns & $*$ & ns & $* *$ & $* *$ & $*$ & ns & ns & $\mathrm{ns}$ \\
\hline 60 & $*$ & ns & $*$ & ns & $* *$ & $* *$ & $* *$ & $* *$ & $* *$ & ns \\
\hline 90 & $* *$ & ns & $*$ & $* *$ & $* *$ & $* *$ & ns & $*$ & $*$ & ns \\
\hline
\end{tabular}

Table Continue...

\begin{tabular}{|c|c|c|c|c|c|c|c|c|c|c|}
\hline \multirow[t]{2}{*}{ Days } & \multicolumn{6}{|c|}{$\mathrm{IAA}+\mathrm{ABA}$} & \multicolumn{4}{|c|}{$\mathrm{IAA}+\mathrm{GA}$} \\
\hline & $\begin{array}{c}\text { Control } \\
\text { vs } 25 \\
\text { mg I I-1 }^{-1} \\
\text { IAA+ABA }\end{array}$ & $\begin{array}{c}\text { Control } \\
\text { vs } 50 \\
\mathrm{mg} \mathrm{l}^{-1} \\
\text { IAA+ABA }\end{array}$ & $\begin{array}{c}\text { Control } \\
\text { vs } 100 \\
\mathrm{mg} \mathrm{l}^{-1} \\
\text { IAA+ABA }\end{array}$ & $\begin{array}{c}25 \mathrm{mg} \mathrm{l}^{-1} \\
\text { IAA+ABA } \\
\text { vs } 50 \\
\mathrm{mg} \mathrm{l}^{-1} \\
\text { IAA+ABA }\end{array}$ & $\begin{array}{c}25 \mathrm{mg} \mathrm{l}^{-1} \\
\text { IAA+ABA } \\
\text { vs } 100 \\
\mathrm{mg} \mathrm{l}^{-1} \\
\text { IAA+ABA }\end{array}$ & $\begin{array}{c}50 \mathrm{mg} \mathrm{l}^{-1} \\
\text { IAA+ABA } \\
\text { vs } 100 \\
\mathrm{mg} \mathrm{l}^{-1} \\
\text { IAA+ABA }\end{array}$ & $\begin{array}{c}\text { Control } \\
\text { vs } 25 \\
\mathrm{mg} \mathrm{l}^{-1} \\
\text { IAA+GA }\end{array}$ & $\begin{array}{c}\text { Control } \\
\text { vs } 50 \mathrm{mg} \\
\mathrm{I}^{-1} \text { IAA+GA }\end{array}$ & $\begin{array}{c}\text { Control } \\
\text { vs } 100 \mathrm{mg} \\
\mathrm{I}^{-1} \text { IAA+GA }\end{array}$ & $\begin{array}{c}25 \mathrm{mg} \mathrm{l}^{-1} \\
\text { IAAGA } \\
\text { vs } 50 \mathrm{mg} \mathrm{l}^{-1} \\
\text { IAA+GA }\end{array}$ \\
\hline 30 & $* *$ & $* *$ & $* *$ & ns & ns & ns & $* *$ & $* *$ & $* *$ & $* *$ \\
\hline 60 & ns & $* *$ & $*$ & $*$ & $* *$ & ns & $*$ & $*$ & $* *$ & $* *$ \\
\hline 90 & ns & $* *$ & $* *$ & ns & $*$ & ns & $* *$ & $* *$ & $* *$ & $* *$ \\
\hline
\end{tabular}

Table Continue...

\begin{tabular}{|c|c|c|c|c|c|c|c|c|}
\hline \multirow[t]{5}{*}{ Days } & \multicolumn{2}{|c|}{$\mathrm{IAA}+\mathrm{GA}$} & \multicolumn{6}{|c|}{$A B A+B A P$} \\
\hline & $25 \mathrm{mg} \mathrm{l}^{-1}$ & $50 \mathrm{mg} \mathrm{l}^{-1}$ & Control & Control & Control & $25 \mathrm{mg} \mathrm{l}^{-1}$ & $25 \mathrm{mg} \mathrm{l}^{-1}$ & $50 \mathrm{mg} \mathrm{l}^{-1}$ \\
\hline & $I A A+G A$ & $\mathrm{IAA}+\mathrm{GA}$ & vs $25 \mathrm{mg} \mathrm{l}^{-1}$ & vs $50 \mathrm{mg} \mathrm{l}^{-1}$ & vs 100 mg & $\mathrm{ABA}+\mathrm{BAP}$ & $A B A+B A P$ & $A B A+B A P$ \\
\hline & vs 100 mg & vs $100 \mathrm{mg} \mathrm{l}^{-1}$ & $\mathrm{ABA}+\mathrm{BAP}$ & $A B A+B A P$ & $\mathrm{I}^{-1} \mathrm{ABA}+\mathrm{BAP}$ & vs $50 \mathrm{mg} \mathrm{l}^{-1}$ & vs $100 \mathrm{mg} \mathrm{l}^{-1}$ & vs $100 \mathrm{mg}$ \\
\hline & $\mathrm{I}^{-1} \mid \mathrm{AA}+\mathrm{GA}$ & $\mathrm{IAA}+\mathrm{GA}$ & & & & $\mathrm{ABA}+\mathrm{BAP}$ & $A B A+B A P$ & $\mathrm{I}^{-1} \mathrm{ABA}+\mathrm{BAP}$ \\
\hline 30 & $* *$ & $* *$ & ns & $*$ & $* *$ & ns & ns & ns \\
\hline 60 & $* *$ & $* *$ & $* *$ & $* *$ & $* *$ & ns & ns & ns \\
\hline 90 & $* *$ & ns & $* *$ & $* *$ & $* *$ & $* *$ & $*$ & ns \\
\hline
\end{tabular}




\begin{tabular}{|c|c|c|c|c|c|c|c|c|c|c|c|c|}
\hline \multirow[t]{2}{*}{ Days } & \multicolumn{6}{|c|}{$\mathrm{BAP}+\mathrm{GA}$} & \multicolumn{6}{|c|}{$A B A+G A$} \\
\hline & $\begin{array}{c}\text { Control } \\
\text { vs } 25 \\
\mathrm{mg} \mathrm{l}^{-1} \\
\text { BAP+ } \\
\text { GA }\end{array}$ & $\begin{array}{c}\text { Control } \\
\text { vs } 50 \\
\mathrm{mg} \mathrm{l}^{-1} \\
\text { BAP+ } \\
\text { GA }\end{array}$ & $\begin{array}{c}\text { Control } \\
\text { vs } 100 \\
\mathrm{mg} \mathrm{l}^{-1} \\
\mathrm{BAP}+ \\
\mathrm{GA}\end{array}$ & $\begin{array}{c}25 \mathrm{mg} / \mathrm{l} \\
\mathrm{BAP}+\mathrm{GA} \\
\mathrm{vs} 50 \\
\mathrm{mg} \mathrm{l}^{-1} \\
\mathrm{BAP}+\mathrm{GA}\end{array}$ & $\begin{array}{c}25 \mathrm{mg} \mathrm{l}^{-1} \\
\mathrm{BAP}+\mathrm{GA} \\
\mathrm{vs} 100 \\
\mathrm{mg} \mathrm{l}^{-1} \\
\mathrm{BAP}+\mathrm{GA}\end{array}$ & $\begin{array}{c}50 \mathrm{mg} \mathrm{l}^{-1} \\
\mathrm{BAP}+\mathrm{GA} \\
\mathrm{vs} 100 \\
\mathrm{mg} \mathrm{l}^{-1} \\
\mathrm{BAP}+\mathrm{GA}\end{array}$ & $\begin{array}{c}\text { Control } \\
\text { vs } 25 \\
\mathrm{mg} \mathrm{l}^{-1} \\
\mathrm{ABA}+ \\
\text { GA }\end{array}$ & $\begin{array}{c}\text { Control } \\
\text { vs } 50 \\
\mathrm{mg} \mathrm{l}^{-1} \\
\mathrm{ABA}+ \\
\mathrm{GA}\end{array}$ & $\begin{array}{c}\text { Control } \\
\text { vs } 100 \\
\mathrm{mg} \mathrm{l}^{-1} \\
\mathrm{ABA}+ \\
\mathrm{GA}\end{array}$ & $\begin{array}{c}25 \mathrm{mg} \mathrm{l}^{-1} \\
\mathrm{ABA}+\mathrm{GA} \\
\text { vs } 50 \\
\mathrm{mg} \mathrm{l}^{-1} \\
\mathrm{ABA}+\mathrm{GA}\end{array}$ & $\begin{array}{c}25 \\
\mathrm{mg} \mathrm{l}^{-1} \\
\mathrm{ABA}+\mathrm{GA} \\
\mathrm{vs} 100 \\
\mathrm{mg} \mathrm{l}^{-1} \\
\mathrm{ABA}+\mathrm{GA}\end{array}$ & $\begin{array}{c}50 \\
\mathrm{mg} \mathrm{l}^{-1} \\
\mathrm{ABA}+\mathrm{GA} \\
\text { vs } 100 \\
\mathrm{mg} \mathrm{l}^{-1} \\
\mathrm{ABA}+\mathrm{GA}\end{array}$ \\
\hline 30 & $* *$ & $*$ & ns & ns & ns & ns & ns & ns & $*$ & ns & ns & ns \\
\hline 60 & $* *$ & ns & ns & ns & $*$ & $*$ & ns & ns & ns & $*$ & $* *$ & ns \\
\hline 90 & ns & ns & ns & ns & $*$ & ns & ns & ns & ns & ns & $*$ & ns \\
\hline
\end{tabular}

${ }^{*} p<0.05,{ }^{* *} p<0.01, * * * p<0.001, \mathrm{~ns}-$ Non-significant $(p>0.05)$

$A B A+G A$, respectively. There was no significant difference between other treated plants.

The effects of phytohormones on morphology of $F$. esculentum revealed that GA and IAA+GA at higher concentration (100 mg $\left.\mathrm{I}^{-1}\right)$ effectively promoted shoot length. There are many reports indicating that application of auxins and gibberellins enhance plant growth (Giannakoula et al., 2012; Ngomuo et al., 2013). Several studies have proved positive effect of GA on shoot length (Giannakoula et al., 2012; Pérez-Jiménez et al., 2015). According to Taiz and Zeiger (2010) gibberellins stimulates cell division and cell elongation which are two events that cause plant elongation. Our results are similar to Naeem et al. (2004) who observed increased shoot length with IAA+GA. The increase in plant height might be due to the stimulatory action of auxin which softens the cell wall by escalating its plasticity or due to oxidative decarboxylation of synthetic auxins which could not be catalyzed by the peroxidase enzyme. Also, the effect of IAA on cell division and cell elongation resulted in increased plant height. Many researchers have observed increased shoot length due to IAA (Mostafa and Abou Al-Hamd, 2011; Muthulakshmi and Pandiyarajan, 2015). In present investigation $A B A$ in combination with IAA $(I A A+A B A)$ and BAP (ABA+BAP) decreased shoot length at higher concentration $\left(50,100 \mathrm{mg} \mathrm{l}^{-1}\right)$. Inhibitory effects of $A B A$ on shoot length was also reported in rice (Chen et al.

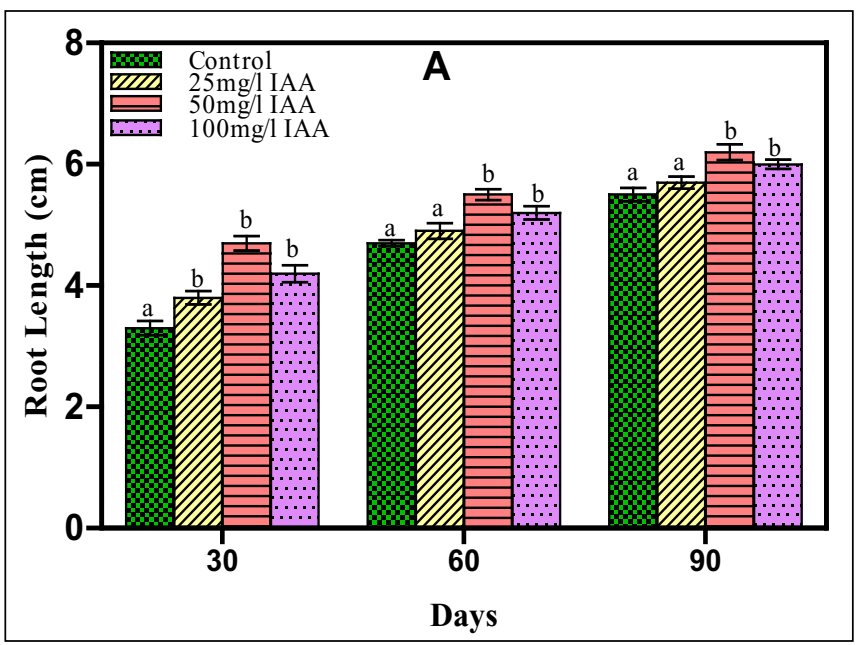

2006; Cha-um et al., 2007), wheat (Zhang and Jiang, 2002) and Cynanchum komarovii (Yang et al. 2007). Similar to our results, Bakrim et al. (2007) observed decreased shoot length in tomato after BAP treatment at higher concentration. IAA application showed decrease in shoot length of lentil (Naeem et al., 2004).

\subsection{Root length}

Root length under phytohormones treatment was observed at 30, 60 and 90 days of growth and the results revealed that there was a progressive increase in root length in plants treated with phytohormones. When plant growth regulators were used solely, IAA $50 \mathrm{mg} \mathrm{l}^{-1}$ treated plants showed maximum root length at 30 days $(4.70 \mathrm{~cm}, 42.4 \%$ increase from control), at 60 days $(5.50 \mathrm{~cm}, 14.5 \%$ increase from control) and at 90 days $(6.20 \mathrm{~cm}, 12.7 \%$ increase from control). BAP $100 \mathrm{mg} \mathrm{l}^{-1}$ treated plants showed minimum root length at 30 days $(2.70 \mathrm{~cm}, 18.2 \%$ decrease from control) and 60 days $(4.10 \mathrm{~cm}, 12.8 \%$ decrease from control). At 90 days BAP $100 \mathrm{mg} \mathrm{l}^{-1}$ and ABA $25 \mathrm{mg} \mathrm{l}^{-1}$ treated plants showed lowest root length $(4.40 \mathrm{~cm}, 20 \%$ decrease from control) (Figure 2). In combined treatment of plant growth regulators, IAA+GA $100 \mathrm{mg} \mathrm{l}^{-1}$ treatment resulted maximum root length at all three intervals of observation. Maximum root length was 5 $\mathrm{cm}$ at 30 days ( $51.5 \%$ increase from control), $5.80 \mathrm{~cm}$ at 60 days (23.4\% increase from control) and $6.60 \mathrm{~cm}$ at 90 days

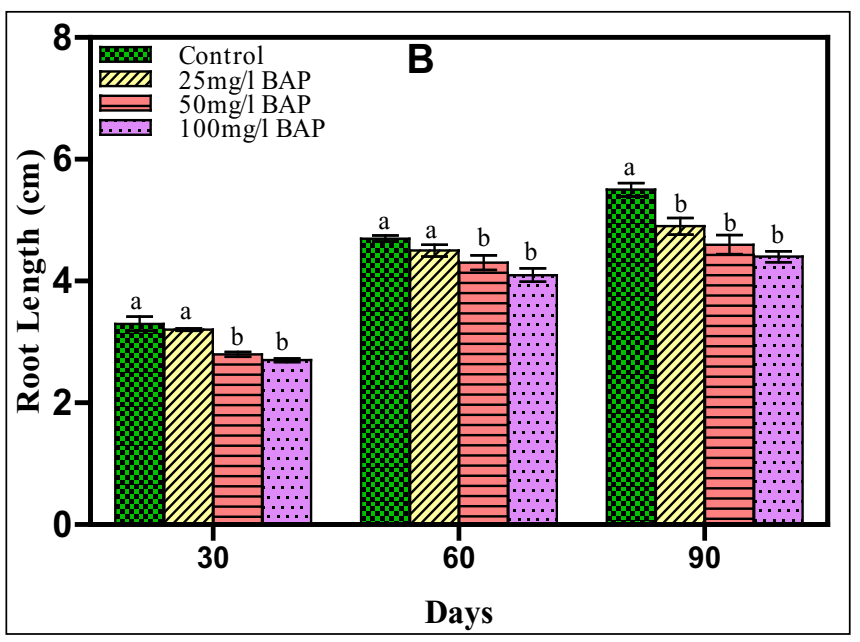

Figure 2: Continue... 

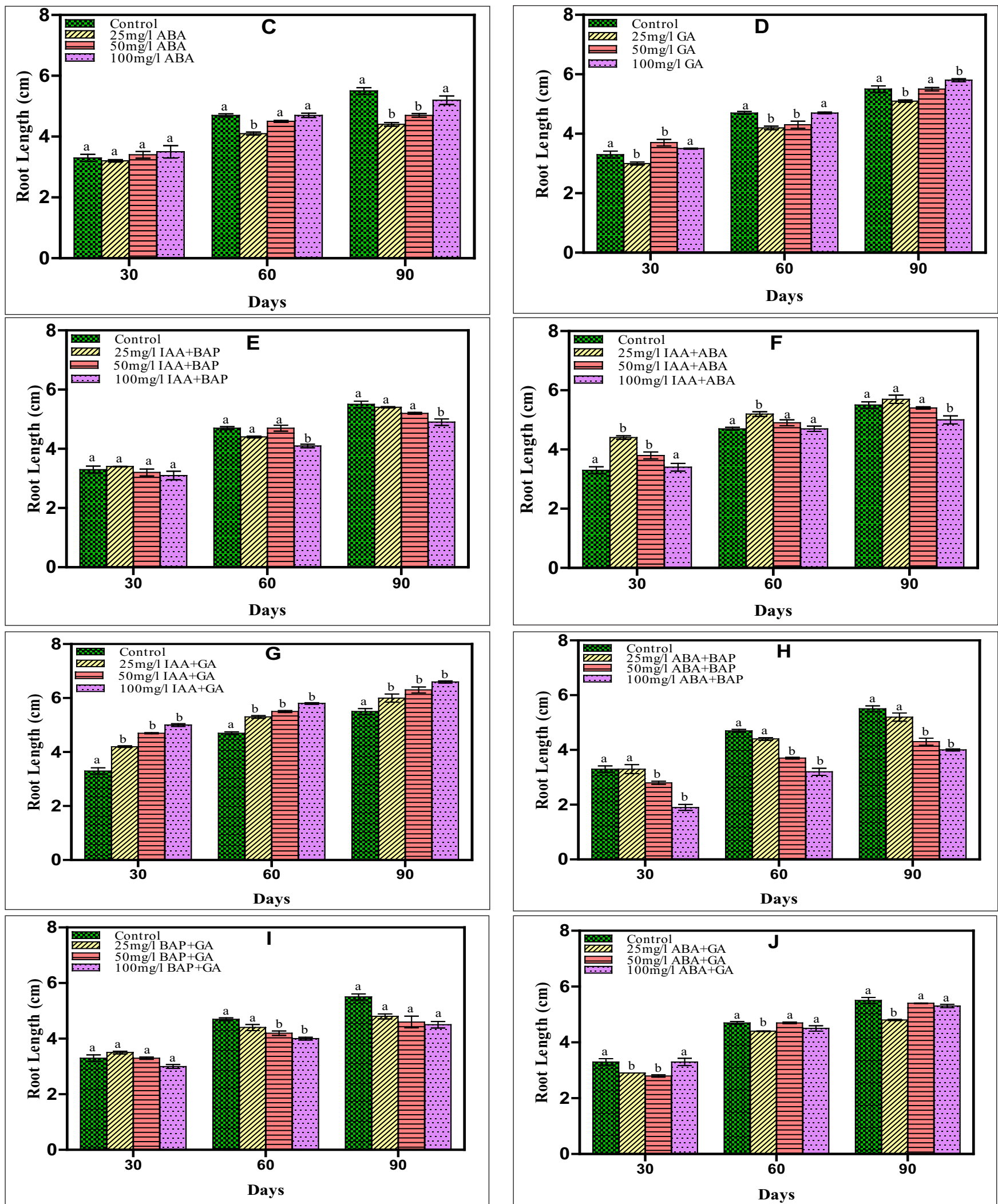

Figure 2: Root length in Fagopyrum esculentum on different days treated with IAA (A), BAP (B), ABA (C), GA (D), IAA+BAP (E), IAA+ABA (F), IAA+GA (G), ABA+BAP (H), BAP+GA (I) and ABA+GA (J). Values are mean $\pm S E ; n=3$, analysed by Two-way ANOVA followed by Bonferroni's multiple comparison test. Values followed by the same letter are not statistically different $(p<0.05)$ compared with control 
( $20 \%$ increase over control). Minimum root length at 30 days $(1.90 \mathrm{~cm}, 42.4 \%$ decrease from control), at 60 days $(3.20 \mathrm{~cm}$, $46.9 \%$ decrease from control) and at 90 days $(4 \mathrm{~cm}, 27.3 \%$ decrease from control) was shown by ABA+BAP $100 \mathrm{mg} \mathrm{l}^{-1}$ treated plants (Figure 2).

It is evident from Figure 2 that growth regulators affected root length compared to untreated plants. In general, IAA (Figure $2 \mathrm{~A}$ ), GA (Figure $2 \mathrm{D}$ ) as well as their combination (Figure $2 \mathrm{G}$ ) and IAA+ABA (Figure $2 \mathrm{~F}$ ) increased root length while $A B A$ (Fig. $2 \mathrm{C}$ ) and BAP (Figure $2 \mathrm{~B}$ ) decreased root length as compared to control. After 90 days Bonferroni post-test revealed (Table
2) significant difference $(p<0.05)$ between untreated (Control) plants and growth regulators $I A A, A B A, B A P, G A, I A A+G A$, $A B A+B A P$ and $B A P+G A$ except $25 \mathrm{mg} \mathrm{l}^{-1} \mathrm{IAA}, 100 \mathrm{mg} \mathrm{l}^{-1} \mathrm{ABA}, 50$ $\mathrm{mg} \mathrm{l}^{-1} \mathrm{GA}, 25 \mathrm{mg} \mathrm{l}^{-1} \mathrm{ABA}+\mathrm{BAP}$ treatments. All the concentration of GA (25, 50 and $\left.100 \mathrm{mg} \mathrm{l}^{-1}\right)$ and IAA+GA (25, 50 and 100 $\mathrm{mg} \mathrm{l}^{-1}$ ) differed significantly with each other. Plants treated with $25 \mathrm{mg} \mathrm{l}^{-1}$ of IAA, ABA+BAP, ABA+GA revealed significant difference with $50 \mathrm{mg} \mathrm{l}^{-1}$ of IAA, ABA+BAP, ABA+GA treated plants. Plants given treatment of $25 \mathrm{mg} \mathrm{l}^{-1} \mathrm{BAP}, \mathrm{ABA}, \mathrm{IAA}+\mathrm{BAP}$, $A B A+B A P, A B A+G A$ was also significant with $100 \mathrm{mg} \mathrm{l}^{-1} B A P$, $A B A, I A A+B A P, A B A+B A P, A B A+G A$. Significant difference was

Table 2: Bonferroni post-test for comparison between treated and untreated plants of Fagopyrum esculentum for root length

\begin{tabular}{|c|c|c|c|c|c|c|c|c|c|c|c|c|}
\hline \multirow[t]{2}{*}{ Days } & \multicolumn{6}{|c|}{ IAA } & \multicolumn{6}{|c|}{ BAP } \\
\hline & $\begin{array}{c}\text { Control } \\
\text { vs } 25 \\
\mathrm{mg} \mathrm{l}^{-1} \\
\text { IAA }\end{array}$ & $\begin{array}{c}\text { Control } \\
\text { vs } 50 \\
\text { mg l}^{-1} \\
\text { IAA }\end{array}$ & $\begin{array}{c}\text { Control } \\
\text { vs } 100 \\
\mathrm{mg} \mathrm{l}^{-1} \\
\text { IAA }\end{array}$ & $\begin{array}{l}25 \mathrm{mg} / \mathrm{I} \\
\text { IAA Vs } \\
50 \mathrm{mg} \\
\mathrm{I}^{-1} \mathrm{IAA}\end{array}$ & $\begin{array}{c}25 \mathrm{mg} \mathrm{l}^{-1} \\
\text { IAA Vs } \\
100 \mathrm{mg} \\
\mathrm{I}^{-1} \text { IAA }\end{array}$ & $\begin{array}{c}50 \mathrm{mg} \mathrm{l}^{-1} \\
\text { IAA Vs } \\
100 \mathrm{mg} \\
\mathrm{I}^{-1} \text { IAA }\end{array}$ & $\begin{array}{l}\text { Control } \\
\text { vs } 25 \\
\text { mg l-1 }^{-1} \\
\text { BAP }\end{array}$ & $\begin{array}{c}\text { Control } \\
\text { vs } 50 \\
\text { mg l-1 }^{-1} \\
\text { BAP }\end{array}$ & $\begin{array}{c}\text { Control } \\
\text { vs } 100 \\
\text { mg l-1 }^{-1} \\
\text { BAP }\end{array}$ & $\begin{array}{c}25 \mathrm{mg} \mathrm{l}^{-1} \\
\text { BAP vs } \\
50 \mathrm{mg} \mathrm{l}^{-1} \\
\text { BAP }\end{array}$ & $\begin{array}{c}25 \mathrm{mg} / \mathrm{l} \\
\text { BAP vs } \\
100 \mathrm{mg} \\
\mathrm{I}^{-1} \mathrm{BAP}\end{array}$ & $\begin{array}{c}50 \mathrm{mg} \mathrm{l}^{-1} \\
\text { BAP vs } \\
100 \mathrm{mg} \\
\mathrm{I}^{-1} \text { BAP }\end{array}$ \\
\hline 30 & $* *$ & $*$ & $* *$ & $*$ & $*$ & $* *$ & ns & $* *$ & $* *$ & $*$ & $* *$ & ns \\
\hline 60 & ns & $*$ & $* *$ & $*$ & ns & ns & ns & $*$ & $*$ & ns & $*$ & ns \\
\hline 90 & ns & $* *$ & $* *$ & $* *$ & ns & ns & $*$ & $*$ & $* *$ & ns & $* *$ & ns \\
\hline
\end{tabular}

Table Continue...

\begin{tabular}{|c|c|c|c|c|c|c|c|c|}
\hline \multirow[t]{2}{*}{ Days } & \multicolumn{6}{|c|}{ ABA } & \multicolumn{2}{|c|}{ GA } \\
\hline & $\begin{array}{c}\text { Control } \\
\text { vs } 25 \mathrm{mg} \mathrm{l}^{-1} \\
\text { ABA }\end{array}$ & $\begin{array}{c}\text { Control } \\
\text { vs } 50 \mathrm{mg} \mathrm{l}^{-1} \\
\text { ABA }\end{array}$ & $\begin{array}{c}\text { Control } \\
\text { vs } 100 \mathrm{mg} \\
\mathrm{I}^{-1} \mathrm{ABA}\end{array}$ & $\begin{array}{c}25 \mathrm{mg} \mathrm{l}^{-1} \mathrm{ABA} \\
\text { vs } 50 \mathrm{mg} \mathrm{l}^{-1} \\
\mathrm{ABA}\end{array}$ & $\begin{array}{c}25 \mathrm{mg} \mathrm{l}^{-1} \\
\mathrm{ABA} \mathrm{Vs} 100 \\
\mathrm{mg} \mathrm{l}^{-1} \mathrm{ABA}\end{array}$ & $\begin{array}{c}50 \mathrm{mg} \mathrm{l}^{-1} \\
\text { ABA Vs } 100 \\
\mathrm{mg} \mathrm{l}^{-1} \mathrm{ABA}\end{array}$ & $\begin{array}{l}\text { Control } \\
\text { Vs } 25 \mathrm{mg} \\
\mathrm{I}^{-1} \mathrm{GA}\end{array}$ & $\begin{array}{l}\text { Control } \\
\text { Vs } 50 \mathrm{mg} \\
\mathrm{I}^{-1} \mathrm{GA}\end{array}$ \\
\hline 30 & ns & ns & ns & ns & ns & ns & $*$ & $* *$ \\
\hline 60 & $*$ & ns & ns & $*$ & $*$ & ns & $*$ & $* *$ \\
\hline 90 & $* *$ & $* *$ & ns & ns & $*$ & $* *$ & $* *$ & ns \\
\hline
\end{tabular}

Table Continue...

\begin{tabular}{|c|c|c|c|c|c|c|c|c|c|c|}
\hline \multirow[t]{2}{*}{ Days } & \multicolumn{4}{|c|}{ GA } & \multicolumn{6}{|c|}{$\mathrm{IAA}+\mathrm{BAP}$} \\
\hline & $\begin{array}{l}\text { Control } \\
\text { vs } 100 \\
\mathrm{mg} \mathrm{l}^{-1} \\
\text { GA }\end{array}$ & $\begin{array}{c}25 \mathrm{mg} \\
\mathrm{I}^{-1} \mathrm{GA} \mathrm{Vs} \\
50 \mathrm{mg} \\
\mathrm{I}^{-1} \mathrm{GA}\end{array}$ & $\begin{array}{c}25 \mathrm{mg} \mathrm{l}^{-1} \\
\mathrm{GA} \mathrm{Vs} \\
100 \mathrm{mg} \\
\mathrm{I}^{-1} \mathrm{GA}\end{array}$ & $\begin{array}{c}50 \mathrm{mg} \\
\mathrm{I}^{-1} \mathrm{GA} \text { vs } \\
100 \mathrm{mg} \\
\mathrm{I}^{-1} \mathrm{GA}\end{array}$ & $\begin{array}{c}\text { Control vs } \\
25 \mathrm{mg} \mathrm{l}^{-1} \\
\text { IAA+BAP }\end{array}$ & $\begin{array}{l}\text { Control } \\
\text { vs } 50 \\
\text { mg l-1 } \\
\text { IAA+BAP }\end{array}$ & $\begin{array}{l}\text { Control } \\
\text { vs } 100 \\
\text { mg l-1 }^{-1} \\
\text { IAA+BAP }\end{array}$ & $\begin{array}{c}25 \mathrm{mg} \mathrm{l}^{-1} \\
\text { IAA+BAP } \\
\text { vs } 50 \mathrm{mg} \mathrm{l}^{-1} \\
\text { IAA+BAP }\end{array}$ & $\begin{array}{c}25 \mathrm{mg} \mathrm{l}^{-1} \\
\text { IAA+BAP } \\
\text { vs } 100 \mathrm{mg} \\
\mathrm{I}^{-1} \mid \mathrm{AA}+\mathrm{BAP}\end{array}$ & $\begin{array}{c}50 \mathrm{mg} \mathrm{l}^{-1} \\
\text { IAA+BAP } \\
\text { vs } 100 \mathrm{mg} \\
\text { I }^{-1} \text { IAA+BAP }\end{array}$ \\
\hline 30 & ns & $* *$ & $*$ & ns & ns & ns & ns & ns & ns & ns \\
\hline 60 & ns & ns & $*$ & $* *$ & ns & ns & $*$ & ns & ns & $*$ \\
\hline 90 & $*$ & $*$ & $* *$ & $*$ & ns & ns & $*$ & ns & $*$ & ns \\
\hline
\end{tabular}

Table Continue...

\begin{tabular}{|c|c|c|c|c|c|c|c|c|c|c|}
\hline \multirow[t]{2}{*}{ Days } & \multicolumn{6}{|c|}{$\mathrm{IAA}+\mathrm{ABA}$} & \multicolumn{4}{|c|}{$\mathrm{IAA}+\mathrm{GA}$} \\
\hline & $\begin{array}{l}\text { Control } \\
\text { vs } 25 \\
\text { mg l}^{-1} \\
\text { IAA+ABA }\end{array}$ & $\begin{array}{l}\text { Control } \\
\text { vs } 50 \\
\mathrm{mg} \mathrm{l}^{-1} \\
\text { IAA+ABA }\end{array}$ & $\begin{array}{l}\text { Control } \\
\text { vs } 100 \\
\mathrm{mg} \mathrm{l}^{-1} \\
\text { IAA+ABA }\end{array}$ & $\begin{array}{c}25 \mathrm{mg} \mathrm{l}^{-1} \\
\text { IAA+ABA } \\
\text { vs } 50 \\
\mathrm{mg} \mathrm{l}^{-1} \\
\text { IAA+ABA }\end{array}$ & $\begin{array}{c}25 \mathrm{mg} \mathrm{l}^{-1} \\
\text { IAA+ABA } \\
\text { vs } 100 \\
\mathrm{mg} \mathrm{l}^{-1} \\
\text { IAA+ABA }\end{array}$ & $\begin{array}{c}50 \mathrm{mg} \mathrm{l}^{-1} \\
\mathrm{IAA}^{-} \mathrm{ABA} \\
\text { vs } 100 \\
\mathrm{mg} \mathrm{l}^{-1} \\
\mathrm{IAA}^{-} \mathrm{ABA}\end{array}$ & $\begin{array}{l}\text { Control } \\
\text { vs } 25 \\
\mathrm{mg} \mathrm{l}^{-1} \\
\text { IAA+GA }\end{array}$ & $\begin{array}{c}\text { Control } \\
\text { vs } 50 \mathrm{mg} \\
\mathrm{I}^{-1} \text { IAA+GA }\end{array}$ & $\begin{array}{c}\text { Control } \\
\text { vs } 100 \mathrm{mg} \\
\mathrm{I}^{-1} \mathrm{IAA}+\mathrm{GA}\end{array}$ & $\begin{array}{c}25 \mathrm{mg} \mathrm{l}^{-1} \\
\text { IAAGA } \\
\text { vs } 50 \mathrm{mg} \mathrm{|}^{-1} \\
\text { IAA+GA }\end{array}$ \\
\hline 30 & $*$ & $* *$ & ns & $* *$ & $*$ & $*$ & $*$ & $* *$ & $*$ & $* *$ \\
\hline 60 & $* *$ & ns & ns & ns & $* *$ & ns & $*$ & $* *$ & $*$ & ns \\
\hline 90 & ns & ns & $* *$ & ns & $*$ & $*$ & $*$ & $*$ & $* *$ & $*$ \\
\hline
\end{tabular}




\begin{tabular}{|c|c|c|c|c|c|c|c|c|c|c|c|c|c|c|c|c|}
\hline \multicolumn{17}{|c|}{ Table Continue... } \\
\hline \multirow[t]{2}{*}{ Days } & \multicolumn{4}{|c|}{$\mathrm{IAA}+\mathrm{GA}$} & \multicolumn{12}{|c|}{$A B A+B A P$} \\
\hline & \multicolumn{2}{|c|}{$\begin{array}{c}25 \mathrm{mg} \mathrm{I}^{-1} \\
\mathrm{IAA}+\mathrm{GA} \\
\text { vs } 100 \mathrm{mg} \\
\mathrm{I}^{-1} \mathrm{IAA}+\mathrm{GA}\end{array}$} & \multicolumn{2}{|c|}{$\begin{array}{c}50 \mathrm{mg} \mathrm{|}^{-1} \\
\mathrm{IAA}+\mathrm{GA} \\
\text { vs } 100 \mathrm{mg} \mathrm{l}^{-1} \\
\mathrm{IAA}+\mathrm{GA}^{-1}\end{array}$} & \multicolumn{2}{|c|}{$\begin{array}{c}\text { Control } \\
\text { vs } 25 \mathrm{mg} \mathrm{l}^{-1} \\
\text { ABA+BAP }\end{array}$} & \multicolumn{2}{|c|}{$\begin{array}{c}\text { Control } \\
\text { vs } 50 \mathrm{mg} \mathrm{l}^{-1} \\
\text { ABA+BAP }\end{array}$} & \multicolumn{2}{|c|}{$\begin{array}{c}\text { Control } \\
\text { vs } 100 \mathrm{mg} \\
\mathrm{I}^{-1} \mathrm{ABA}+\mathrm{BAP}\end{array}$} & \multicolumn{2}{|c|}{$\begin{array}{c}25 \mathrm{mg} \mathrm{l}^{-1} \\
\text { ABA+BAP } \\
\text { vs } 50 \mathrm{mg} \mathrm{l}^{-1} \\
\text { ABA+BAP }\end{array}$} & \multicolumn{2}{|c|}{$\begin{array}{c}25 \mathrm{mg} \mathrm{l}^{-1} \\
\text { ABA+BAP } \\
\text { vs } 100 \mathrm{mg} \mathrm{l}^{-1} \\
\text { ABA+BAP }\end{array}$} & \multicolumn{2}{|c|}{$\begin{array}{c}50 \mathrm{mg} \mathrm{l}^{-1} \\
\mathrm{ABA}+\mathrm{BAP} \\
\text { vs } 100 \mathrm{mg} \\
\mathrm{l}^{-1} \mathrm{ABA}+\mathrm{BAP}\end{array}$} \\
\hline 30 & \multicolumn{2}{|c|}{$*$} & \multicolumn{2}{|c|}{$*$} & \multicolumn{2}{|l|}{ ns } & \multicolumn{2}{|r|}{$* *$} & \multicolumn{2}{|l|}{$*$} & \multicolumn{2}{|c|}{$* *$} & \multicolumn{2}{|l|}{$*$} & \multicolumn{2}{|r|}{$*$} \\
\hline 60 & \multicolumn{2}{|l|}{$*$} & \multicolumn{2}{|r|}{$*$} & \multicolumn{2}{|l|}{ ns } & \multicolumn{2}{|r|}{$*$} & \multicolumn{2}{|l|}{$*$} & \multicolumn{2}{|r|}{$*$} & \multicolumn{2}{|l|}{$*$} & \multicolumn{2}{|r|}{$* *$} \\
\hline 90 & \multicolumn{2}{|l|}{$*$} & & $*$ & \multicolumn{2}{|l|}{ ns } & \multicolumn{2}{|r|}{$*$} & \multicolumn{2}{|l|}{$* *$} & & $* *$ & \multicolumn{2}{|l|}{$* *$} & \multicolumn{2}{|r|}{ ns } \\
\hline \multicolumn{17}{|c|}{ Table Continue... } \\
\hline Days & & & & & $P+G A$ & & & & & & & $A B$ & $A+G A$ & & & \\
\hline & $\begin{array}{c}\text { Control } \\
\text { vs } 25 \\
\mathrm{mg} \mathrm{l}^{-1} \\
\text { BAP+ } \\
\text { GA }\end{array}$ & $\begin{array}{r}\text { Con } \\
\text { vs } \\
\text { me } \\
\text { BA } \\
\text { G }\end{array}$ & $\begin{array}{l}\text { trol } \\
50 \\
\mathrm{I}^{-1} \\
\mathrm{P}+ \\
\mathrm{A}\end{array}$ & $\begin{array}{c}\text { Control } \\
\text { vs } 100 \\
\mathrm{mg} \mathrm{l}^{-1} \\
\text { BAP+ } \\
\text { GA }\end{array}$ & $\begin{array}{c}25 \mathrm{mg} / \mathrm{l} \\
\mathrm{BAP}+\mathrm{GA} \\
\text { vs } 50 \\
\mathrm{mg} \mathrm{l}^{-1} \\
\mathrm{BAP}+\mathrm{GA}\end{array}$ & $\begin{array}{r}25 \\
\text { BAF } \\
\text { vs } \\
\text { m } \\
\text { BAP }\end{array}$ & $\begin{array}{l}g^{-1} \\
-G A \\
00 \\
I^{-1} \\
-G A\end{array}$ & $\begin{array}{c}50 \mathrm{mg} \mathrm{l}^{-1} \\
\mathrm{BAP}+\mathrm{GA} \\
\text { vs } 100 \\
\mathrm{mg} \mathrm{l}^{-1} \\
\mathrm{BAP}+\mathrm{GA}\end{array}$ & $\begin{array}{c}\text { Control } \\
\text { vs } 25 \\
\mathrm{mg} \mathrm{l}^{-1} \\
\mathrm{ABA}+ \\
\mathrm{GA}\end{array}$ & $\begin{array}{c}\text { Con } \\
\text { vs } \\
\mathrm{mg} \\
\mathrm{AB} \\
\mathrm{G}\end{array}$ & $\begin{array}{l}\text { trol } \\
50 \\
\mathrm{I}^{-1} \\
\mathrm{~A}+ \\
\mathrm{A}\end{array}$ & $\begin{array}{c}\text { Control } \\
\text { vs } 100 \\
\mathrm{mg} \mathrm{l}^{-1} \\
\mathrm{ABA}+ \\
\mathrm{GA}\end{array}$ & $\begin{array}{c}25 \mathrm{mg} \mathrm{l}^{-1} \\
\mathrm{ABA}+\mathrm{GA} \\
\mathrm{vs} 50 \\
\mathrm{mg} \mathrm{l}^{-1} \\
\mathrm{ABA}+\mathrm{GA}\end{array}$ & $\begin{array}{r}2 \\
\mathrm{~m} \\
\mathrm{ABA} \\
\mathrm{vs} \\
\mathrm{m} \\
\mathrm{ABA}\end{array}$ & $\begin{array}{l}5 \\
f^{-1} \\
+G A \\
100 \\
l^{-1} \\
+G A\end{array}$ & $\begin{array}{c}50 \\
\mathrm{mg} \mathrm{l}^{-1} \\
\mathrm{ABA}+\mathrm{GA} \\
\mathrm{vs} 100 \\
\mathrm{mg} \mathrm{l}^{-1} \\
\mathrm{ABA}+\mathrm{GA}\end{array}$ \\
\hline 30 & ns & $\pi$ & IS & ns & ns & & & ns & * & $*$ & * & ns & ns & & 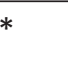 & $*$ \\
\hline 60 & ns & & * & $*$ & ns & & & ns & $*$ & $\mathrm{n}$ & & ns & $*$ & & s & ns \\
\hline 90 & $* *$ & & * & $*$ & ns & & & ns & $* *$ & $\mathrm{n}$ & $\mathrm{s}$ & ns & $*$ & & 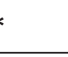 & ns \\
\hline
\end{tabular}

${ }^{*} p<0.05, * * p<0.01, * * * p<0.001$, ns-Non-significant $(p>0.05)$

seen in $50 \mathrm{mg} \mathrm{l}^{-1}$ of $A B A$ and $I A A+A B A$ with; $100 \mathrm{mg} \mathrm{l}^{-1}$ of $A B A$ and $I A A+A B A$, respectively. Non-significant variations were detected in rest of the treatments. Auxin increase rooting by stimulation of division of primer root cells. Auxins usually stimulate formation of root and balance morphogenesis such as shoot and root development (Rout et al., 2000). In the present study, root length was influenced positively by IAA at $50 \mathrm{mg} \mathrm{l}^{-1}$ and IAA+GA at $100 \mathrm{mg} \mathrm{l}^{-1}$ concentration. Similar results regarding effect of IAA on root length has been reported (Alam et al., 2012; Muthulakshmi and Pandiyarajan, 2015). Ghodrat et al. (2013) in a study also reported increased root length after application of GA on Oryza sativa. In present studies decrease in root length was seen in ABA+BAP (100 mg $\left.\mathrm{I}^{-1}\right)$. Earlier reports have also shown decrease in root length due to ABA (Liao et al., 2008; Cutler et al., 2010). Our results are in agreement with Bakrim et al. (2007) who reported that BAP inhibited root elongation in tomato.

\section{Conclusion}

Need for food and medicines is supposed to continue due to ever-growing world population. Exploitation of 'underutilized' crops can contribute effectively to promote nourishment and biological sustainability. Fagopyrum esculentum Moench is one of the essential neglected crops having high nutritive and medicinal value. From the present study it can be concluded that PGRs effectively increased shoot and root length of Fagopyrum esculentum. Combination of PGRs were more effective than solely applied PGRs. The results of the present study call for further research on mechanism of PGRs action by using molecular approaches.

\section{References}

Ahmada, T., Zia-ul-Hassanb, S.M., Iqbalc, Q., Ghanib, M.A., Tariqd, R.M., 2019. Effect of PGRs on Vegetative and Reproductive Traits of Bitter Gourd (Momordica charantia L.): A Field Assessment. Journal of Horticultural Science and Technology 2(2), 41-44.

Alam, M.M., Naeem, M., Idrees, M., Khan, M.M.A., 2012. Moinuddin. Augmentation of photosynthesis, crop productivity, enzyme activities and alkaloids production in Sadabahar (Catharanthus roseus L.) through application of diverse plant growth regulators. Journal of Crop Science and Biotechnology 15(2), 117-129.

Amri, E., 2011. The effect of auxins (IBA, NAA) on vegetative propagation of medicinal plant Bobgunnia madagascariensis (Desv.) JH Kirkbr and Wiersema. Tanzania Journal of Natural and Applied Sciences 2(1), 359-66.

Bakrim, A., Lamhamdi, M., Sayah, F., Chib, F., 2007. Effects of plant hormones and 20-hydroxyecdysone on tomato (Lycopersicum esculentum) seed germination and seedlings growth. African Journal of Biotechnology 6(24), 2792-2802.

Cha-um, S., Roytakul, S., Sathung, T., Maijandang, A., Kirdmane, C., 2007. Effect of exogenous glucose and abscisic acid on physiological and morphological 
performances of in vitro Indica rice (Oryza sativa L. sp. indica). American Journal of Plant Physiology 2, 155-66.

Chen, C.W., Yang, Y.W., Lur, H.S., Tsai, Y.G., Chang, M.C., 2006. A novel functions of abscisic acid in the regulation of rice (Oryza sativa L.) root growth and development. Plant and Cell Physiology 47(1), 1-3.

Cutler, S.R., Rodriguez, P.L., Finkelstein, R.R., Abrams, S.R., 2010. Abscisic acid: emergence of a core signaling network. Annual Review of Plant Biology 61, 651-679.

Fabjan, N., Rode, J., Kosir, I.J., Zhang, Z., Kreft, I., 2003. Tartary buckwheat (Fagopyrum tartarikum Gaertn.) as a source of dietary rutin and quercetin. Journal of Agricultural and Food Chemistry. 51, 6452-6455.

Galavi, M., Karimian, M.A., Mousavi, S.R., 2013. Effects of different auxin (IBA) concentrations and planting-beds on rooting grape (Vitis vinifera L.) cuttings. Annual Review and Research in Biology 3(4), 517-523.

Ghodrat, V., Moradshahi, A., Rousta, M.J., Karampour, A., 2013. Improving yield and yield components of rice (Oryza sativa L.) by indolebutyric acid (IBA), gibberellic acid $\left(\mathrm{GA}_{3}\right)$ and salicylic acid (SA) pre-sowing seed treatments. American-Eurasian Journal of Agricultural and Environmental Sciences 13(6), 872-876.

Giannakoula, A.E., Ilias, I.F., Maksimović, J.J., Maksimović, V.M., Zivanovic, B.D., 2012. The effects of plant growth regulators on growth, yield and phenolic profile of lentil plants. Journal of Food Composition and Analysis 28(1), 46-53.

Gulpinar, A.R., Orhan, I.E., Kan, A., Senol, F.S., Celik, S.A., Kartal, M., 2012. Estimation of in vitro neuroprotective properties and quantification of rutin and fatty acids in buckwheat (Fagopyrum esculentum Moench) cultivated in Turkey. Food research international 46(2), 536-543.

Khunte, S.D., Kumar, A., Ansari, N., Saravanan, S., 2020. Effect of Different Levels of PGRs with Organic Manure on Growth Characters and Economics of Strawberry (Fragariaxananassa Duch.) cv. Chandler in Northern region. International Journal of Current Microbiology and Applied Sciences 9(1), 1633-1638.

Liao, Y., Zhang, J.S., Chen, S., Zhang, W., 2008. Role of soybean GmbZIP132 under abscisic acid and salt stresses. Journal of Integrative Plant Biology 50, 221-230.

Morishita, T., Yamaguchi, H., Degi, K., 2007. The contribution of polyphenols to antioxidative activity in common buckwheat and tartary buckwheat grain. Plant Production Science 10, 99-104.

Mostafa, G.G., Abou Alhamd, M.F., 2011. Effect of gibberellic acid and indole- 3 acetic acid on improving growth and accumulation of phytochemical composition in Balanites aegyptica plants. American Journal of Plant Physiology 6, 36-43.
Muthulakshmi, S., Pandiyarajan, V., 2015. Effect of IAA on the Growth, Physiological and Biochemical Characteristics in Catharanthus roseus (L). G. Don. S. International Journal of Science and Research 4(3), 442-448.

Naeem, M., Bhatti, I.R., Ahmad, R.H., Ashraf, M.Y., 2004. Effect of some growth hormones ( $\mathrm{GA}_{3}$, IAA and Kinetin) on the morphology and early or delayed initiation of bud of lentil (Lens culinaris medik). Pakistan Journal of Botany 36(4), 801-809.

Ngomuo, M., Mneney, E., Ndakidemi, P., 2013. The effects of auxins and cytokinin on growth and development of (Musa sp.) var. "Yangambi" explants in tissue culture. American Journal of Plant Sciences 4(11), 2174-2180.

Pérez-Jiménez, M., Pazos-Navarro, M., López-Marín, J., Gálvez, A., Varó, P., del Amor, F.M., 2015. Foliar application of plant growth regulators changes the nutrient composition of sweet pepper (Capsicum annuum L.). Scientia Horticulturae 194, 188-93.

Rout, G.R., Samantaray, S., Das, P., 2000. In vitro manipulation and propagation of medicinal plants. Biotechnology Advances 18, 91-120.

Sharma, A.J., Puri, S., Bhattacharya, Randev, N.D., 2018. Drought Stress-Mediated Consequences on Enzymatic Antioxidants of Fagopyrum esculentum Moench. Asian Journal of Advanced Basic Sciences 6(1), 22-27.

Stibilj, V., Kreft, I., Smrkolj, P., Osvald, J., 2004. Enhanced selenium content in buckwheat (Fagopyrum esculentum Moench) and pumpkin (Cucurbita pepo L.) seeds by foliar fertilisation. European Food Research and Technology 219(2), 142-144.

Sumathi, A., Prasad, V.B., Vanangamudi, M., 2017. Influence of plant growth regulators on yield and yield components in pigeonpea. Legume Research-an International Journal 40(04).

Taiz, L., Zeiger, E., 2010. Plant physiology 5th Ed. Sunderland, MA: Sinauer Associates.

Wijngaard, H.H., Arendt, E.K., 2006. Buckwheat. Cereal Chemistry 83(4), 391-401.

Yang, L., Yu, C.L., Shi, F., Wei, Y.Q., Wang, C.C., Hu, H.T., et al., 2007. Effects of abscisic acid on growth and dehydration tolerance of Cynanchum komarovii seedlings. Plant Growth Regulation 51(2), 177-184.

Zhang, J., Jiang, M., 2002. Involvement of plasma-membrane NADPH oxidase in abscisic acid and water stress-induced antioxidant defense in leaves of maize seedlings. Planta 215, 1022-1030.

Zhang, Z.L., Zhou, M.L., Tang, Y., Li, F.L., Tang, Y.X., Shao, J.R. et al., 2012. Bioactive compounds in functional buckwheat food. Food Research International 49(1), 389-395. 